\title{
Study of momentum transfers within a vegetation canopy
}

\author{
MahendRa Mohan* and M K TIWARI \\ Radio and Atmospheric Science Division, National Physical Laboratory, New Delhi 110 012, India. \\ *e-mail:mmohan@mail.nplindia.ernet.in
}

Two models have been developed by applying conditions of continuity between the roughness sublayer and the top of vegetation canopy having constant foliage distribution. Massman's cosh-type of wind profile and Albini's exponential wind profile have been used to derive expressions for shear stress, displacement height and roughness length in analytical forms. The computed results compared with those of Massman models $(1987,1997)$ show similarity with the present models.

\section{Introduction}

Modeling of momentum transfers by the vegetation to the atmosphere is of great importance in view of applications to agriculture, forestry and atmospheric circulation at the mesoscale and the global scale. The proper quantification of the transfer of $\mathrm{CO}_{2}$, water vapour, ozone and other trace gases to and from vegetation canopies requires an understanding of the structure of atmospheric turbulence within and above vegetation canopy.

Simple parameterizations of vegetations roughness length $\left(z_{0}\right)$ and displacement height $(d)$ as functions of canopy height $(h)$ and leaf area index (LAI) play a key role in the modeling of atmospheric-biospheric exchange process. From the analytical model of wind profiles and shear stress within canopies of arbitrary foliage distribution and parameterization of friction velocity $\left(u_{*}\right)$, one can determine $d / h$ and $z_{0} / h$ as a function of drag area index.

Massman (1987) used a semi-analytical method introducing cosh-type of wind profile model to describe the mean wind profile and shear stress within plant canopy and thus estimated the roughness length and displacement height. The method incorporated the density and vertical structure of the canopy with the parameterization of roughness sub-layer and shelter factor. In this way, Massman (1987) compared several wind profile models against observed data.
Massman (1997) again used Albini's model of wind profile (1981) to develop one dimensional analytical model of momentum transfer and parameterized the surface drag coefficient to estimate $d / h$ and $z_{0} / h$ as functions of foliage structure and foliage density.

The present paper deals with the Massman (1987) model and Albini's model (Massman 1997). Both these models have been redefined in association with the roughness sub-layer existing at the top of the canopy for the case of constant foliage density under neutral condition. Thus, identifying two regimes of flow: one within the vegetation canopy and another above the top of vegetation canopy i.e., roughness sub-layer, the exact analytical expressions for normalized shear stress, displacement height and roughness length have been presented. Some computed results are compared with those of two models in order to test the validity of the expressions worked out.

\section{Mathematical formulations}

Momentum transfer within a vegetation canopy for the first order closure (Sellers et al 1986 and Massman 1987) is described by

$$
\tau=\rho K(z) \frac{d u}{d z}
$$

Keywords. Wind profile; roughness sub-layer; shear stress; displacement height; roughness length; drag coefficient; shelter effect. 


$$
\frac{d \tau}{d z}=\rho C_{d} a(z) u^{2}
$$

where $K$ is the turbulent diffusivity, $u$ is the mean horizontal wind speed, $\tau$ is the shear stress within the canopy, $C_{d}$ is the drag coefficient, $z$ is the height above the ground surface, $\rho$ is the density of air, and $a(z)$ is the foliage distribution or foliage area density (one sided leaf area per unit volume of canopy).

Assuming similarity between wind speed and eddy diffusivity profiles within the canopy with the following dimensionless variables:

$$
\xi=\frac{z}{h}, \chi=\frac{u^{2}}{u_{h}^{2}}, \text { and } \frac{K}{h u}=\frac{K_{h}}{h u_{h}}=\sigma,
$$

the equations (1) and (2) can be coupled together into the form:

$$
\frac{d^{2} \chi}{d \xi^{2}}=\beta^{2} f(\xi) \chi,
$$

where $h$ is the height of the canopy, $u_{h}$ is the mean horizontal speed at the top of canopy, $K_{h}$ is the eddy diffusivity at the top of canopy, $\sigma$ is an unknown proportionality constant and $\beta$ is the profile extinction coefficient defined as:

$$
\begin{aligned}
\beta & =\sqrt{\frac{2 C_{d} \mathrm{LAI}}{\mu \sigma}} \\
\text { and } \mu & =\int_{0}^{1} f(\xi) d(\xi),
\end{aligned}
$$

where $f(\xi)$ is $a(z)$ normalized by the maximum value of foliage area density.

\subsection{Roughness sub-layer}

Raupach et al (1991) and Cellier and Brunet (1992) have explained the role of the atmospheric layer closest to a vegetative surface and referred to it as 'the roughness sub-layer'. This layer is unlike the inertial (or logarithmic) layer because it is associated with multiple scale lengths. In this layer there is a significant departure of the wind profile from that predicted by the logarithmic relationship, giving the values greater than observed (Wilson et al 1982; Shaw and Pereira 1982; Sellers et al 1986).

This problem is comprehensively considered by Garratt (1980); Raupach and Thom (1981). They have noted that the estimates of momentum transfer coefficient $K$ above a vegetative surface were 1.5-2.0 times greater than the value given by the simple logarithmic relationship within the vegetation. The modified simple logarithmic relation to account for the flow in the roughness sub-layer is given by

$$
u(z)=\frac{u_{*}}{\alpha k} \ln \frac{z-d}{z_{0}},
$$

where $\alpha$ is the dimensionless constant estimated to be between 1.5 and 2.0 (Raupach and Thom 1981; Massman 1987), $k$ is the von Karman constant taken to be 0.41 .

The corresponding equation for eddy diffusivity in the roughness sub-layer is also written as:

$$
K(z)=\alpha k u_{*}(z-d) .
$$

\subsection{Cosh-type model}

Massman (1987) has discussed three possible solutions to the equation (3) but adopted the following wind profile for a constant foliage distribution within the canopy

$$
\frac{u}{u_{h}}=\sqrt{\frac{\cosh \beta \xi}{\cosh \beta}} .
$$

Now identifying two regimes of flow: one within the canopy represented by equation (8), and another above the canopy i.e., the roughness sub-layer described by equation (6), we can write the following expressions for win speed $u$ and eddy diffusivity $K$ within and above canopy under the neutral conditions:

$$
\begin{gathered}
u_{1}(z)=\frac{u_{*}}{\alpha k} \ln \frac{z-d}{z_{0}} \quad \text { for } z \geq h \\
u(z)=u_{2}(z)=u_{h} \sqrt{\frac{\cosh \beta\left(\frac{z}{h}\right)}{\cosh \beta}} \quad \text { for } h \geq z \geq 0,
\end{gathered}
$$

and

$$
\begin{gathered}
K_{1}(z)=\alpha k u_{*}(z-d) \text { for } z \geq h \\
K(z)=K_{2}(z)=\sigma h u_{h} \sqrt{\frac{\cosh \beta\left(\frac{z}{h}\right)}{\cosh \beta}} \text { for } h \geq z \geq 0,
\end{gathered}
$$

where the subscripts 1 and 2 denote the flow in the roughness sub-layer and within the canopy.

The following conditions of continuity yield the relations:

$$
\begin{aligned}
& \text { (a) } u_{1}(h)=u_{2}(h): \quad \frac{u_{*}}{\alpha k} \ln \frac{h-d}{z_{0}}=u_{h}, \\
& \text { (b) }\left(\frac{\partial u_{1}}{\partial z}\right)_{h}=\left(\frac{\partial u_{2}}{\partial z}\right)_{h}: \\
& \frac{u_{*}}{\alpha k}(h-d)^{-1}=\frac{1}{2} u_{h} \frac{\beta}{h} \tanh \beta,
\end{aligned}
$$


(c) $\quad K_{1}(h)=K_{2}(h): \quad \alpha k u_{*}(h-d)=\sigma h u_{h}$.

After combining the above relations, the expressions for $u_{*}(z) / u_{h}, u_{*}(h) / u_{h}, d / h$, and $z_{0} / h$ can be obtained as:

$$
\begin{aligned}
\frac{u_{*}^{2}(z)}{u_{h}^{2}} & =\frac{1}{2} \sigma \beta \frac{\sinh \beta\left(\frac{z}{h}\right)}{\cosh \beta}, \\
\frac{u_{*}^{2}(h)}{u_{h}^{2}} & =\frac{1}{2} \sigma \beta \tanh \beta \\
\frac{d}{h} & =1-\frac{1}{\alpha k} \sqrt{\frac{2 \sigma}{\beta \tanh \beta}}, \\
\frac{z_{0}}{h} & =\frac{1}{\alpha k} \sqrt{\frac{2 \sigma}{\beta \tanh \beta}} \exp \left[-\alpha k \sqrt{\frac{2}{\sigma \beta \tanh \beta}}\right] .
\end{aligned}
$$

\subsection{Albini's model}

Albini (1981) modelled the wind speed within the canopy as an exponential function of cumulative leaf drag area per unit planform area

$$
\frac{u(z)}{u_{h}}=e^{-n\left\{1-\frac{\zeta(z)}{\zeta(h)}\right.},
$$

where $\xi$ is the cumulative leaf drag area per unit planform area:

$$
\zeta(z)=\int_{0}^{z}\left[\frac{C_{d}\left(z^{\prime}\right) a\left(z^{\prime}\right)}{P_{m}\left(z^{\prime}\right)}\right] d z^{\prime}
$$

and $\zeta(h)$ is the drag area index. Here $P_{m}(z)$ is the foliage shelter factor for momentum as a function of height within the canopy.

Using Albini's model, Massman (1997) formulated an analytical one-dimensional model of momentum transfer and has shown that

- if the product $\left[C_{d}(z) a(z) / P_{m}(z)\right]$ is uniform within the canopy, then the equation (14) reduces to the exponential canopy wind profile, $\exp \{-n(1-z / h)\}$ and

- if $C_{d}(z)$ is uniform throughout the canopy and if the effect of sheltering is ignored then $\zeta(h)$ reduces to $C_{d} \mathrm{LAI}$.

Massman (1997) parameterized the surface drag coefficient $C_{\text {surf }}=2 u_{*}^{2} / u(h)^{2}$ from which the exponential power $n$ can be expressed as

$$
n=\frac{\zeta(h)}{2\left[c_{1}-c_{2} e^{-c_{3} \zeta(h)}\right]^{2}},
$$

where $c_{1}=0.320, c_{2}=0.264$ and $c_{3}=15.1$ are constants.
Again applying the conditions of continuity as described in the previous section, the expressions for the shear stress and the normalized displacement height and roughness length are found to be

$$
\begin{aligned}
\frac{u_{*}^{2}(z)}{u_{h}^{2}} & =\sigma n \frac{C_{d}(h) a(h)}{P_{m}(h)} \frac{h}{\zeta(h)} e^{-2 n\left(1-\frac{\zeta(z)}{\zeta(h)}\right)}, \\
\frac{u_{*}^{2}(h)}{u_{h}^{2}} & =\sigma n \frac{C_{d}(h) a(h)}{P_{m}(h)} \cdot \frac{h}{\zeta(h)}, \\
\frac{d}{h} & =1-\frac{1}{\alpha k} \sqrt{\frac{\sigma}{n} \frac{\zeta(h)}{h} \frac{P_{m}(h)}{C_{d}(h) a(h)}}, \\
\frac{z_{o}}{h} & =\frac{1}{\alpha k} \sqrt{\frac{\sigma}{n} \frac{\zeta(h)}{h} \frac{P_{m}(h)}{C_{d}(h) a(h)}} \\
& \exp \left[-\alpha k \sqrt{\frac{1}{\sigma n} \frac{\zeta(h)}{h} \frac{P_{m}(h)}{C_{d}(h) a(h)}}\right] .
\end{aligned}
$$

\section{Results and discussions}

We have carried out computations for the present model with the values used by Massman (1987) for cosh-type of wind model of constant foliage distribution. Figure 1 shows the normalized cosh-type canopy wind profile for $C_{d} \mathrm{LAI}=0.6$ which is same as figure 1 of Massman model (1987). We have shown in figure 2 the corresponding shear stress profile within the canopy which is in full agreement with that of Massman's model (1987, figure 2). It

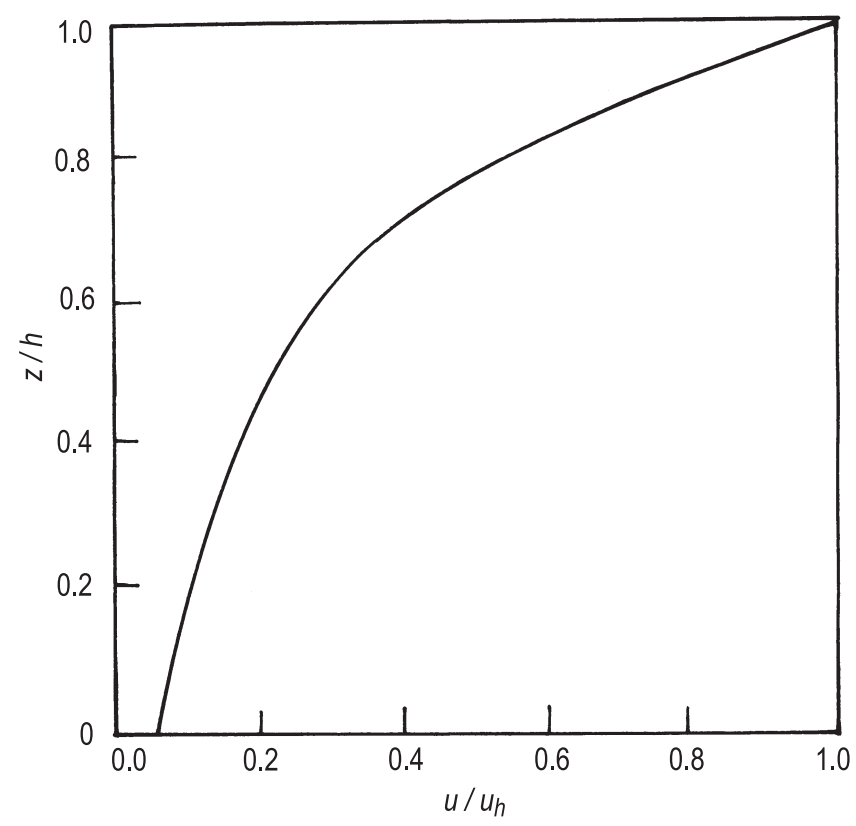

Figure 1. Normalized wind profile for $C_{d} \mathrm{LAI}=0.6$. 


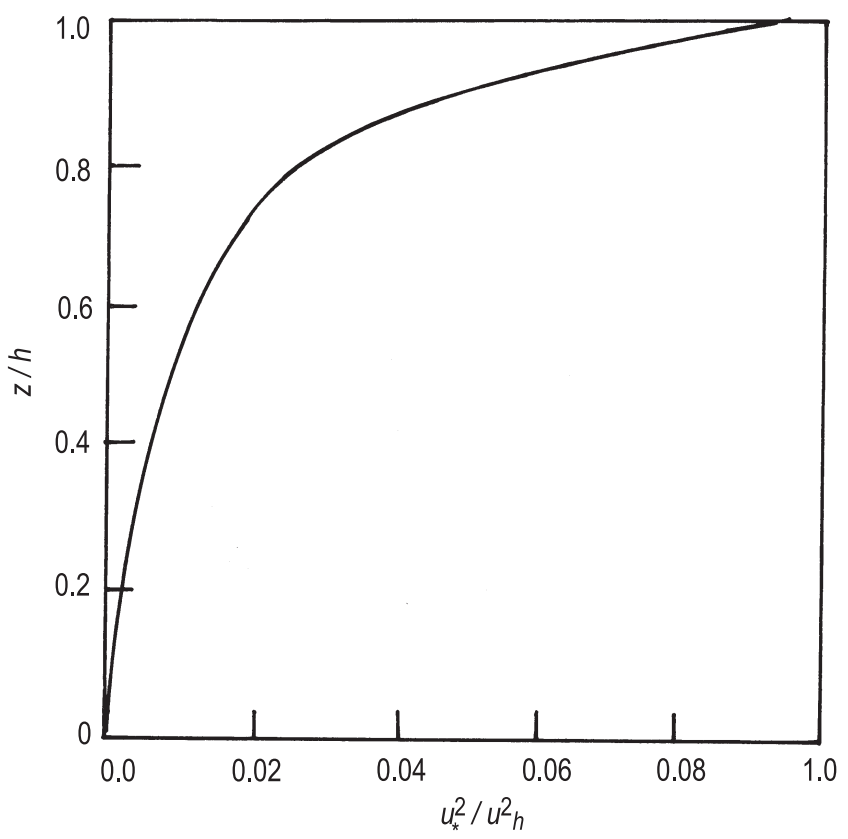

Figure 2. Canopy shear stress profile corresponding to that shown in figure 1.

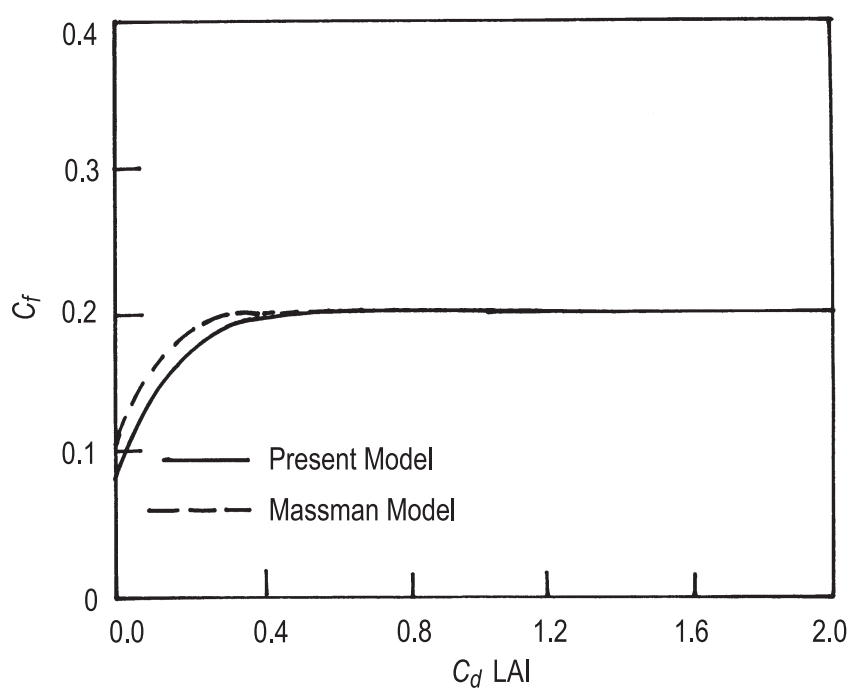

Figure 3. Stand drag coefficient, $C_{f}=2\left(u_{*} / u_{h}\right)^{2}$, as a function of $C_{d} \mathrm{LAI}$.

can be noted from equation (10) that the shear stress within the canopy is independent of the presence of roughness sub-layer. Figure 3 shows stand drag coefficient, $C_{f}=2\left(u_{*}(h) / u_{h}\right)^{2}$ as a function of $C_{d}$ LAI. The solid curve represents the present model while the dotted curve represents the Massman's model (1987). Both the profiles are quite similar.

The normalized displacement height $d / h$ and the roughness length $z_{0} / h$ as a function of $C_{d} \mathrm{LAI}$ are shown in figures 4 and 5 respectively. The solid curve represents the present model while the dotted curve represents Massman's model (1987). From

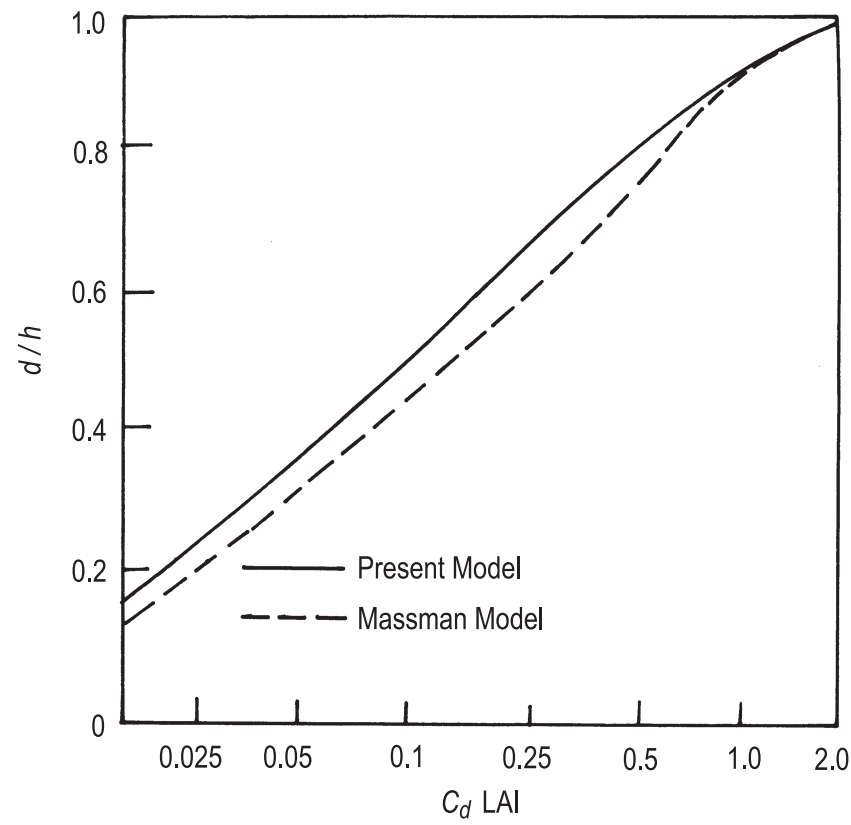

Figure 4. Normalized displacement height, $d / h$, as a function of $C_{d} \mathrm{LAI}$.

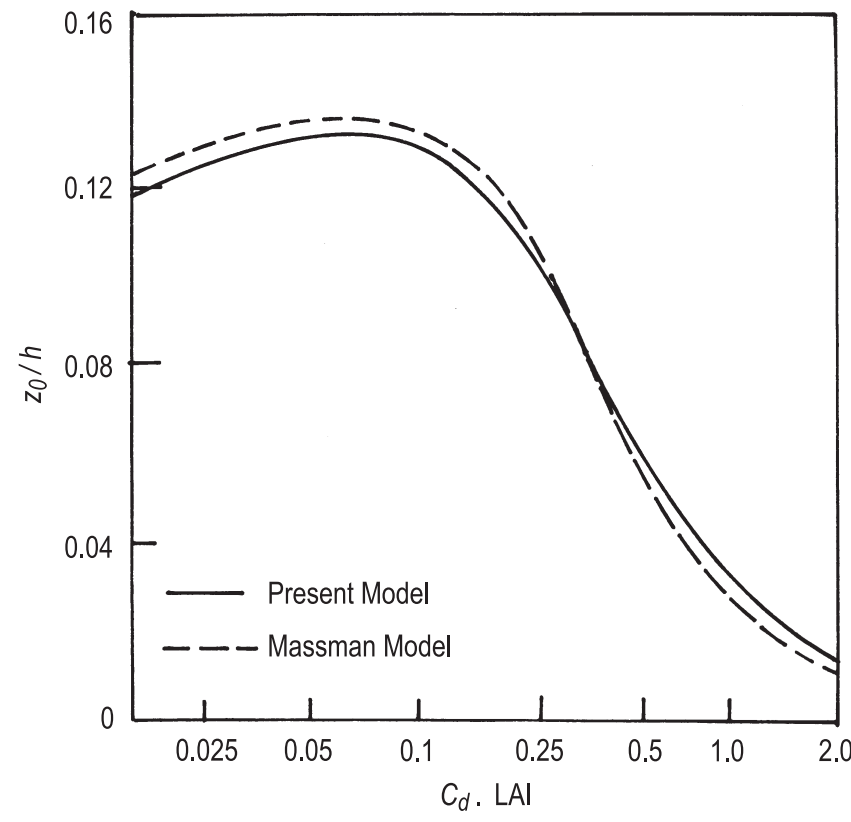

Figure 5. Normalized roughness length, $z_{0} / h$, as a function of $C_{d} \mathrm{LAI}$.

figures 4 and 5 , it can be seen that both the models produce similar $d / h$ and $z_{0} / h$ profiles.

Figure 6 displays $z_{0} / h$ versus $(1-d / h)$. The profile so obtained is in full agreement with that of Massman's model (1987) for $\alpha=1.5$. As $\alpha$ increases, slope of the curve and its maximum peak value also increase (Massman 1987).

The canopy wind profile given by the Albini model (1981) is a generalization of the exponential wind profile. We have presented some com- 


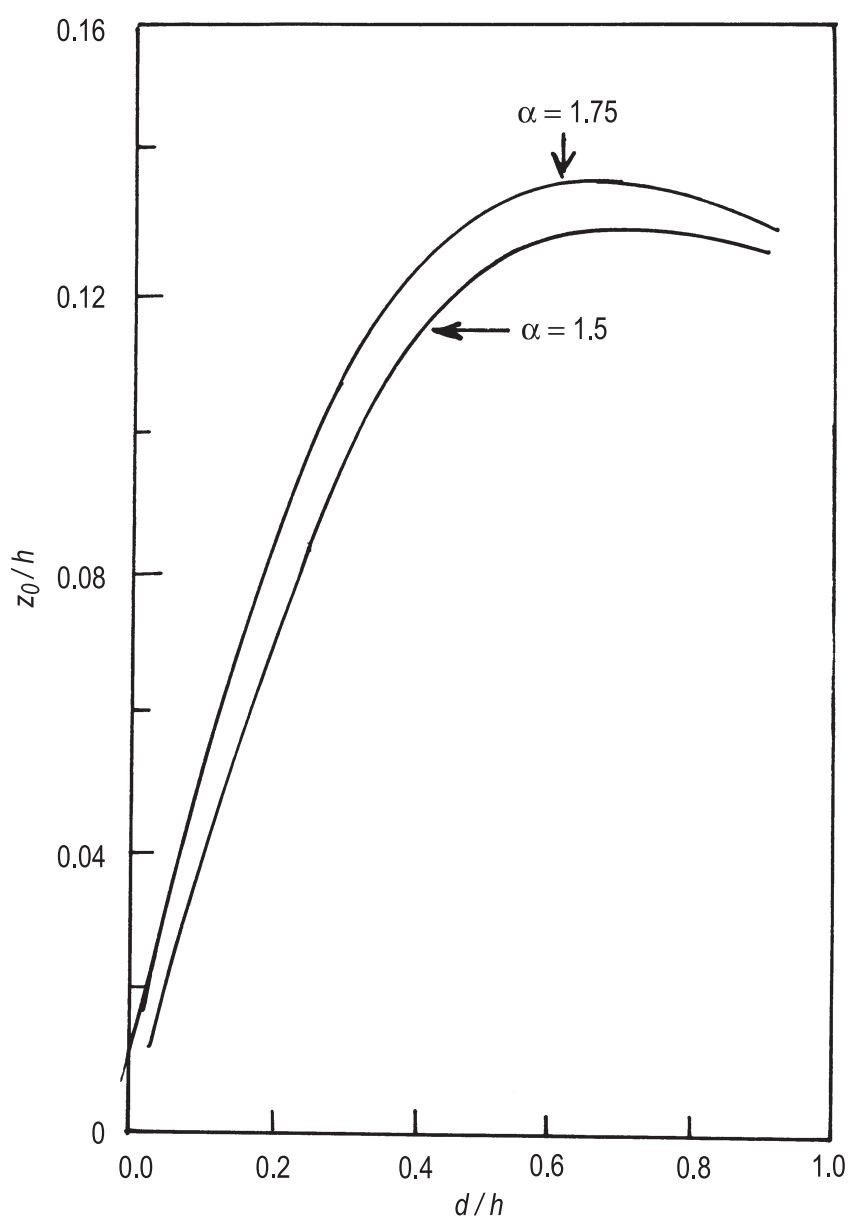

Figure 6. Normalized roughness length, $z_{0} / h$, versus $(1-d / h)$ for different values of $\alpha$.

puted estimates of $d / h$ and $z_{0} / h$ and compared them with those of Massman's model (1997) for constant foliage distribution. We have used modified beta distribution (Massman 1982) to describe the canopy of height-varying foliage density. Since the proportionality constant $\sigma$ is unknown, it can be determined from the given surface drag coefficient profile within the canopy. From the surface drag profile given by Massman (1997, figure 1), we have used equation (18) to estmate $\sigma$ which comes out to be 0.34. $\alpha$ is kept at 1.67 (Massman 1987). Here we note from equations (19) and (20) that both the displacement height $d / h$ and roughness length $z_{0} / h$ depend upon the values of drag coefficient $C_{d}(h)$ and the shelter factor $P_{m}(h)$ at the top of the canopy.

We have shown in figures 7 and 8 the normalized displacement height $d / h$ and roughness length $z_{0} / h$ as a function of drag area index $\zeta(h)$ for constant foliage distribution. We have chosen the profile of Massman model (1997, figures 5 and 6) denoted by dotted curve for $C_{d}(h)=C_{d}(1)=0.2, \alpha_{2}=0$. Comparison shows similarity between the present model and the Massman's model (1997).

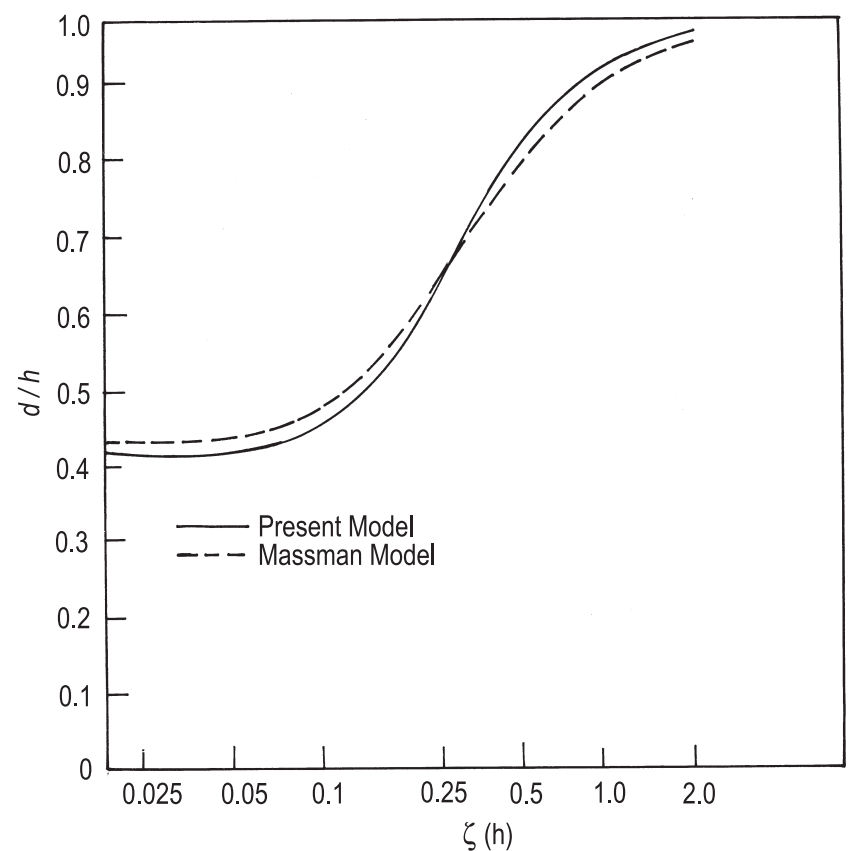

Figure 7. Normalized canopy displacement height, $d / h$, as a function of drag area index, $\zeta(h)$, for drag coefficient $C_{d}(h)=0.2$ with $\alpha_{2}=0$.

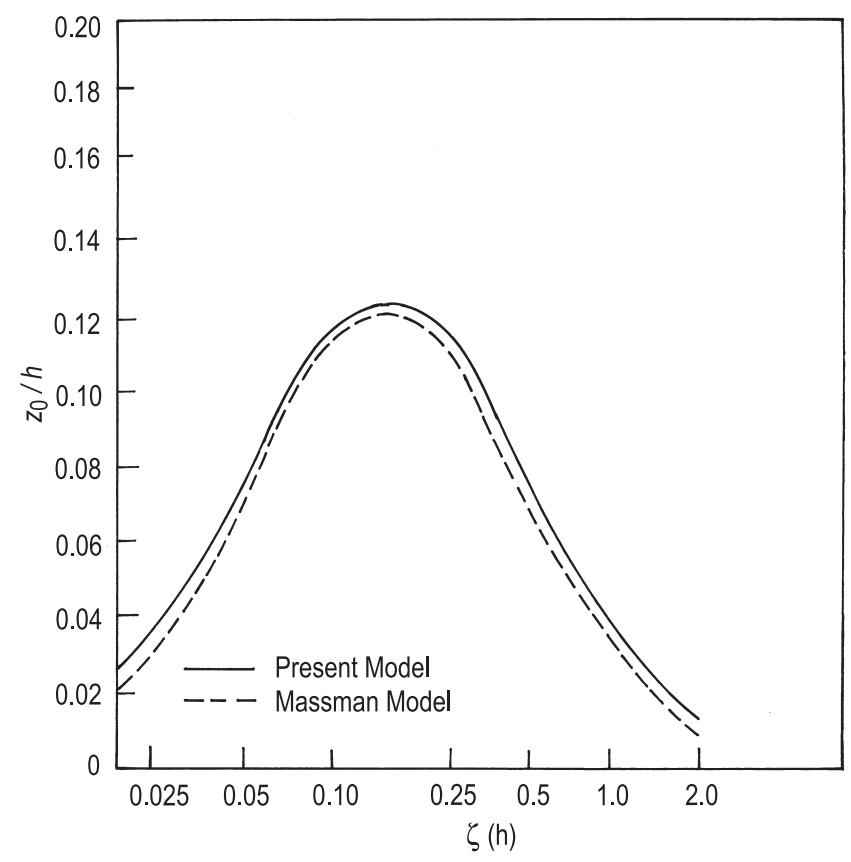

Figure 8. Normalized canopy roughness length, $z_{0} / h$, as a function of drag area index, $\zeta(h)$, for drag coefficient $C_{d}(h)=0.2$ with $\alpha_{2}=0$.

Again figures 9 and 10 show the normalized displacement height $d / h$ and roughness length $z_{0} / h$ as a function of drag area index $\zeta(h)$ for constant foliage distribution under shelter effect. We have taken profile from Massman model (1997, figures 7 and 8) denoted by dotted curve with $P_{m}(h)=$ $\left(1+\alpha_{1} h a(h)\right)$ and $\alpha_{1}=0.4$. It is evident from fig- 


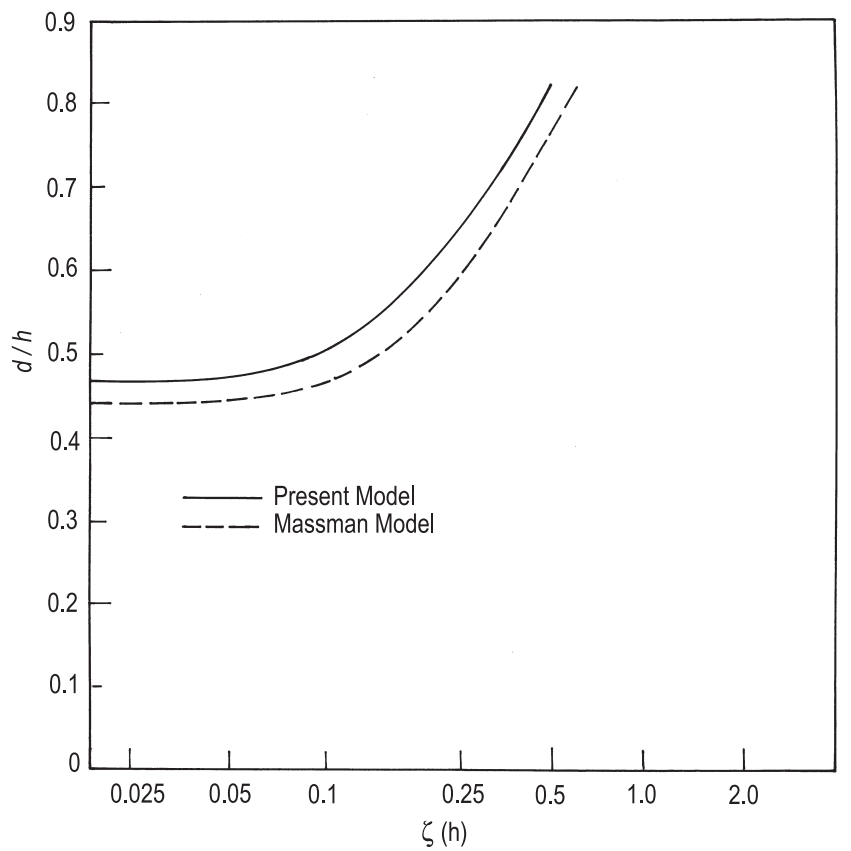

Figure 9. Normalized canopy displacement height, $d / h$, as a function of drag area index, $\zeta(h)$, with the shelter factor $P_{m}=\left(1+\alpha_{1} h a(h)\right)$ and $\alpha_{1}=0.4$.

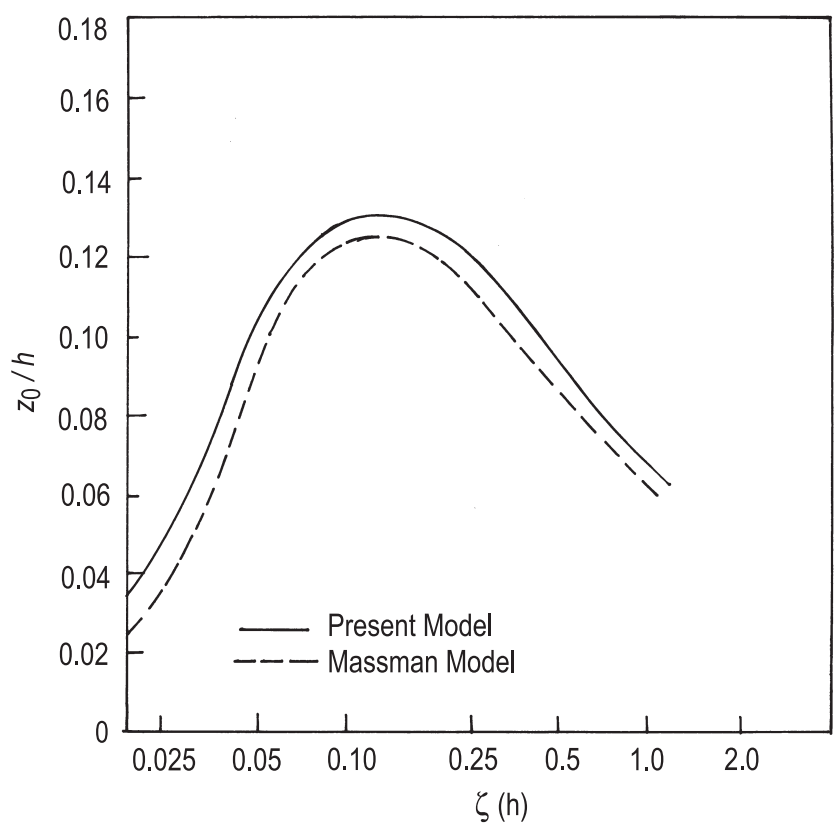

Figure 10. Normalized canopy roughness length, $z_{0} / h$, as a function of drag area index, $\zeta(h)$, with the shelter factor $P_{m}=\left(1+\alpha_{1} h a(h)\right)$ and $\alpha_{1}=0.4$.

ures 9 and 10 that both the models produce the similar profiles with a minor difference that the present model gives a little higher $d / h$ and $z_{0} / h$.
In the first review of this paper, Dr. W J Massman very kindly pointed out the typographical mistake lying in his paper (1997). In his paper the shelter effect is given by the relation $P_{m}(h)=$ $1 /\left(1+\alpha_{1} h a(h)\right)$ while the correct one is $P_{m}(h)=$ $\left(1+\alpha_{1} h a(h)\right)$. Dr. Massman took pains to check his original computer code and found that the calculations were done with $P_{m}(h)=1+\alpha_{1} h a(h)$.

The simple analytical models developed in this study are based on the conditions of continuity at the interface between the inertial sub-layer and the top of the canopy for the case of constant foliage distribution. The simple analytical expressions for shear stress, displacement height and roughness length within the canopy are derived using Massman's cosh-type of wind profile (1987) and Albini's wind profile (1981). The computed results show a fairly good similarity to those of Massman's models $(1987,1997)$. The models thus presented can be used to estimate $d / h$ and $z_{0} / h$ which are useful in land-surface parameterizations for use in global climate models.

\section{References}

Albini F A 1981 A phenomenological model for wind speed and shear stress profiles in vegetation cover layers; $J$. Appl. Meteorol. 20 1325-1335

Cellier P and Brunet Y 1992 Flux gradient relationship above tall plant canopies; Agric. For. Meteorol. 58 93117

Garratt J R 1980 Surface influence upon vertical profiles in the atmospheric near surface layer; Q. J. R. Meteorol. Soc. 106 803-819

Massman W J 1982 Foliage distribution in old-growth coniferous tree canopies; Can. J. Forest Res. 12 10-17

Massman W J 1987 A comparative study of some mathematical models of mean wind structure and aerodynamic drag of plant canopies; Boundary Layer Meteorology 40 179-197

Massman W J 1997 An analytical one-dimensional model of momentum transfer by the vegetation of arbitrary structure; Boundary Layer Meteorology 83 407-421

Raupach M R, Antonia R A and Rajgopalan S 1991 Rough wall turbulent boundary layers; Appl. Mech. Rev. 44 $1-25$

Raupach M R and Thom A S 1981 Turbulence in and above plant canopies; Ann. Rev. Fluid Mech. 13 97-129

Sellers P J, Mintz Y, Sud Y C and Dachler A 1986 A simple biospheric model $(\mathrm{SiB})$ for the use in general circulation models; J. Atmos. Sci. 43 505-531

Shaw R H and Pereira A R 1982 Aerodynamic roughness of plant canopy: A numerical experiment; Agric. Meteorol. 26 51-65

Wilson J D, Ward D P, Thurtell G W and Kidd G E 1982 Statistics of atmospheric turbulence within and above corn canopy; Boundary Layer Meteorology $\mathbf{2 4}$ 495-519 